\title{
Binary Star Research Using the MACHO Database
}

\author{
Kem H. Cook \\ Lawrence Livermore National Laboratory, 7000 East Avenue, \\ Livermore, CA 94550, USA
}

The MACHO Collaboration has produced a variable star catalog of the Magellanic Clouds which is a rich source of eclipsing binary systems. I will review the techniques that have been used by MACHO for identifying eclipsing binaries in a relatively automated fashion which is necessitated by the more than 230000 variable light curves cataloged. We have identified about 6000 eclipsing binaries using these techniques. These systems have periods ranging from hours to more than 1000 days. I will survey the binary star data from MACHO and highlight its use in investigating topics spanning the physics of supersoft gamma-ray sources to the distance of the Large Magellanic Cloud and point to future directions of research using this public resource. 